Bull. Austral. Math. Soc.

Vol. 45 (1992) [201-213]

\title{
LATTICE ISOMORPHISMS OF ORTHODOX SEMIGROUPS
}

\author{
KATHERINE G. JohNSTON
}

To the memory of Professor F.D. Cleary

\begin{abstract}
It is shown that the set of all orthodox subsemigroups of an orthodox semigroup forms a lattice. This lattice is a join-sublattice of the lattice of all semigroups, but is not in general a meet-sublattice. We obtain results concerning lattice isomorphisms between orthodox semigroups, several of which include known results for inverse semigroups as special cases.
\end{abstract}

\section{INTRODUCTION}

Lattices of subalgebras have long been studied for various types of algebras, for example groups, semigroups, completely simple semigroups, and inverse semigroups. (See, for instance the surveys [15] and [16].) However, as was pointed out in [9], the set of all regular subsemigroups of a regular semigroup does not form a lattice in any natural way. We show here that for orthodox semigroups the partially ordered set of regular (orthodox) subsemigroups of an orthodox semigroup $S$ does form a lattice, although it is not a sublattice of the lattice of all subsemigroups of $S$.

In Section 3 we consider lattice isomorphisms between orthodox semigroups, and show that modular inverse semigroups are strictly lattice determined within the class of orthodox semigroups.

In Section 4, lattice isomorphisms of simple and 0 -simple orthodox semigroups are studied in detail. We show that modular inverse semigroups are lattice determined within the class of orthodox semigroups. The class of simple orthodox semigroups whose non-zero idempotents do not form a left-zero or right-zero band is seen to be latticeclosed. These results include several known results for inverse semigroups as special cases.

Received 7 March 1991

The author wishes to thank T.E. Hall for many enlightening conversations on orthodox semigroups.

Copyright Clearance Centre, Inc. Serial-fee code: 0004-9729/92 \$A2,00+0.00. 


\section{Preliminaries}

For a semigroup $S$, we shall denote by $\mathcal{L}(S)$ the lattice of all subsemigroups of $S$. If $S$ is an orthodox semigroup, $\mathcal{L O}(S)$ will mean the set of all orthodox (equivalently, regular) subsemigroups of $S$. Clearly $\mathcal{L O}(S) \subseteq \mathcal{L}(S)$, and $\mathcal{L O}(S)$ is partially ordered under set inclusion. Also, $\mathcal{L O}(S)$ has a greatest element and a least element, $S$ and $\phi$, respectively.

THEOREM 1. Take any orthodox semigroup $S$, and let $\left\{S_{i}: i \in I\right\}$ be any subset of $\mathcal{L O}(S)$. Then the join, $\vee\left\{S_{i}: i \in I\right\}$, is an orthodox subsemigroup of $S$.

Proof: It suffices to show that $T=\vee\left\{S_{i}: i \in I\right\}$ is regular. Take any $x \in T$, say $x=x_{i_{1}} x_{i_{2}} \ldots x_{i_{n}}$, where $x_{i_{k}} \in S_{i_{k}}$. Each $x_{i_{k}}$ has an inverse $x_{i_{k}}^{\prime}$ in $S_{i_{k}}$, and by [13], $x_{i_{n}}^{\prime} x_{i_{n-1}}^{\prime} \ldots x_{i_{1}}^{\prime} \in V(x)$, so $x$ has an inverse in $T$.

COROLLARY 2. Let $S$ be an orthodox semigroup. Then $\mathcal{L O}(S)$ is a complete join-subsemilattice of $\mathcal{L}(S)$.

The following is an elementary result from lattice theory. (See, for example [3].)

RESULt 3. Let $P$ be a partially ordered set with least and greatest element. If every subset of $P$ has a least upper bound in $P$, then $P$ is a complete lattice.

Combining this with Theorem 1 we get the following.

Theorem 4. Let $S$ be an orthodox semigroup. Then $\mathcal{L O}(S)$ is a complete lattice.

From Theorem 4 we know that any subset of $\mathcal{L O}(S)$ has a meet; Theorem 6 below gives a description of the meet. The meet in $\mathcal{L}(S)$ is set intersection. We give an example to show that this is not the case in $\mathcal{L O}(S)$.

Example. Let $S=\mathcal{M}^{0}(\{0,1\},\{e\},\{0,1,2\} ; P)$ where $P=\left[\begin{array}{ll}e & 0 \\ 0 & e \\ e & 0\end{array}\right]$ and $\{e\}$ is the trivial group. Then $A=\mathcal{M}^{0}(\{0,1\},\{e\},\{0,1\} ; Q)$ and $B=\mathcal{M}^{\dot{0}}(\{0,1\},\{e\},\{1,2\} ; R)$ where

$$
Q=\left[\begin{array}{ll}
e & 0 \\
0 & e
\end{array}\right] \quad \text { and } \quad R=\left[\begin{array}{ll}
0 & e \\
e & 0
\end{array}\right]
$$

are regular subsemigroups of $S$, but $A \cap B=\mathcal{M}^{0}(\{0,1\},\{e\},\{1\} ;[0 e])$ which is not regular. The meet of $A$ and $B$ in $\mathcal{L O}(S)$ is the one element band $\mathcal{M}^{0}(\{1\},\{e\},\{1\} ;[e])$.

In order to characterise the meet in $\mathcal{L O}(S)$ we use the following result due to T.E. Hall [5, Result 7]. For a semigroup $S$, let $\operatorname{Reg}(S)$ denote the set of regular elements of $S$.

RESULT 5. The regular elements of a semigroup $S, \operatorname{Reg}(S)$, form a subsemigroup if and only if the product of any two idempotents of $S$ is a regular element. 
We shall use the symbol $\wedge$ for the meet operation in $\mathcal{L O}(S)$. This will not cause confusion since set intersection is the meet in $\mathcal{L}(S)$ and we shall consistently use $\cap$ in that case.

THEOREM 6. Let $A$ and $B$ be orthodox subsemigroups of the orthodox semigroup $S$. Then $A \wedge B=\operatorname{Reg}(A \cap B)$.

Proof: Since $A \wedge B \subseteq A$ and $A \wedge B \subseteq B$, clearly $A \wedge B \subseteq A \cap B$, and since $A \wedge B$ is regular, $A \wedge B \subseteq \operatorname{Reg}(A \cap B)$.

Now $\operatorname{Reg}(A \cap B) \subseteq A$ and $\operatorname{Reg}(A \cap B) \subseteq B$. Also $\operatorname{Reg}(A \cap B) \in \mathcal{L O}(S)$, by Result 5, and hence $\operatorname{Reg}(A \cap B) \subseteq A \wedge B$.

\section{LATTICE ISOMORPHISMS}

We shall say that two orthodox semigroups $S$ and $T$ are lattice isomorphic if there is an isomorphism $\Phi$ of $\mathcal{L O}(S)$ onto $\mathcal{L O}(T)$. A mapping $\alpha$ from $S$ to $T$ is said to induce $\Phi$ if $A \Phi=A \alpha$ for all $A$ in $\mathcal{L O}(S)$.

Notice that if $S$ is an inverse semigroup, then $\mathcal{L O}(S)$ is just the lattice of inverse subsemigroups of $S$. Call an inverse semigroup $S$ strongly (lattice) determined by $\mathcal{L O}(S)$ within the class of orthodox semigroups $\mathcal{K}$ if every lattice isomorphism from $S$ to an orthodox semigroup $T$ in $\mathcal{K}$ is induced by an isomorphism of $S$ onto $T$. In this section we shall show that the bicyclic semigroup $\mathcal{B}$ is strongly lattice determined within the class of orthodox semigroups. If we restrict ourselves to the class of inverse semigroups, we obtain the known result that $\mathcal{B}$ is strongly lattice determined within the class of inverse semigroups.

First an example will show that an orthodox semigroup which is not inverse may be lattice isomorphic to an inverse semigroup. Thus the class of inverse semigroups is not necessarily lattice closed.

EXAmple 7. Let $S=\{e, f\}$ be the two-element semilattice, and let $T=\{g, h\}$ be the two-element right-zero semigroup. Clearly, $\mathcal{L O}(S) \cong \mathcal{L O}(T)$, but $S$ is not isomorphic to $T$.

Take any two orthodox semigroups $S$ and $T$ such that $\mathcal{L O}(S)$ is isomorphic to $\mathcal{L O}(T)$ under the (lattice) isomorphism $\Phi$, say. The atoms of $\mathcal{L O}(S)$ are precisely those orthodox subsemigroups $\{e\}$ where $e$ is in $E_{S}$. Thus $\Phi$ induces a one-to-one correspondence $\alpha$ from $E_{S}$ to $E_{T}$, and $E_{S} \Phi=\left(\vee\left\{\{e\}: e \in E_{S}\right\}\right) \Phi=\vee\{\{e\} \Phi: e \in$ $\left.E_{S}\right\}=E_{T}$.

Lemma 8. Suppose $S$ and $T$ are orthodox semigroups, $\Phi$ a lattice isomorphism from $\mathcal{L O}(S)$ onto $\mathcal{L O}(T)$, and $\alpha$ the induced bijection from $E_{S}$ onto $E_{T}$. Let $e, f \in$ $E_{S}$. Then $\{e, f\}$ is a subsemigroup of $S$ (equivalently, $e \mathcal{R} f, e \mathcal{L} f$, or $e$ is comparable to $f$ ) if and only if $\{e \alpha, f \alpha\}$ is a subsemigroup of $T$. 
Proof: Suppose $\langle e, f\rangle=\{e, f\}$. Thus

$$
\begin{aligned}
\langle e \alpha, f \alpha\rangle & =\langle e \alpha\rangle \vee\langle f \alpha\rangle \\
& =(\langle e\rangle \Phi) \vee(\langle f\rangle \Phi) \\
& =(\langle e\rangle \vee\langle f\rangle) \Phi \\
& =\{e, f\} \Phi .
\end{aligned}
$$

Now $\{e, f\}$ covers precisely the atoms $\{e\}$ and $\{f\}$ in $\mathcal{L O}(S)$ so $\{e, f\} \Phi$ covers precisely the atoms $\{e\} \Phi=\{e \alpha\}$ and $\{f\} \Phi=\{f \alpha\}$ in $\mathcal{L O}(T)$, whence $\{e, f\} \Phi=$ $\{e \alpha, f \alpha\}$, and so $\{e \alpha, f \alpha\}$ is a subsemigroup of $T$. That $\Phi^{-1}$ is a lattice isomorphism of $T$ onto $S$ gives us the reverse implication.

Recall that the band $E_{T}$ is a semilattice $Y$ of rectangular bands $V(e), e \in E_{T}$. (See, for example [7, Chapter VI].) Hall [6] defined a band $E$ to be almost commutative provided that $a b=b a$ for all $a, b$ in $E$ where $(a, b) \notin \mathcal{D}^{E}$.

LEMma 9. If $\mathcal{L O}(S) \cong \mathcal{L O}(T)$ and $E_{S}$ is a chain and $e \in E_{T}$, then

(i) $V(e)$ is either a left-zero or right-zero semigroup,

(ii) the structure semilattice of $E_{T}$ is a chain, and

(iii) $E_{T}$ is almost commutative.

Proof: (i) By way of contradiction, suppose that $V(e)$ has two $\mathcal{R}$-classes and two $\mathcal{L}$-classes. Then there are distinct elements $e, f, g, h \in V(e)$ such that $e \mathcal{R} f \mathcal{L} h \mathcal{R} g$. By Lemma $8, f$ and $g$ are comparable elements in a rectangular band, a contradiction.

(ii) Take any $e, f \in E_{T}$ such that $V(e) \neq V(f)$. By Lemma 8, $e$ and $f$ are comparable, and thus $V(e)$ and $V(f)$ are comparable.

(iii) If $e, f \in E_{T}$ and $(e, f) \notin \mathcal{D}^{E_{T}}$, then $(e, f) \notin \mathcal{R}$ and $(e, f) \notin \mathcal{L}$, whence $e$ and $f$ are comparable. Thus $e f=f e$.

Lemma 10. (Jones [11, Lemma 2.1]). If $U$ and $V$ are proper inverse subsemigroups of the bicyclic semigroup $\mathcal{B}$ such that $U \vee V=\mathcal{B}$, then $U \cap V \neq \emptyset$.

The technical lemma below generalises [11, Lemma 2.2].

Lemma 11. Suppose $s$ is an orthodox semigroup such that $E_{S}$ is a semilattice $Y$ of rectangular bands $V(e), e \in E_{S}$, and $E_{S}$ is almost commutative. If, for each $e \in E_{S}, V(e)$ is a left-zero or right-zero semigroup and $Y$ is a chain, then any union of $\mathcal{D}^{S}$-classes is an orthodox subsemigroup.

Proof: Take any collection $\left\{D_{i}: i \in I\right\}$ of $\mathcal{D}^{S}$-classes of $S$, and set $D=$ $\bigcup\left\{D_{i}: i \in I\right\}$. Select any $x, y \in D$, say $x \in D_{i}, y \in D_{j}$. Pick $x^{\prime} \in V(x)$ and $y^{\prime} \in V(y)$.

Case 1: Suppose $x^{\prime} x \mathcal{R} y y^{\prime}$. Then $x^{\prime} x \in R_{y} \cap L_{x}$, and hence $x y \in R_{x} \cap L_{y}$ (see [2, Theorem 2.17]), $x y \mathcal{D} x$, that is $x y \in D_{i}$. 
Case 2: If $x^{\prime} x \mathcal{L} y y^{\prime}$, a proof analogous to case 1 will suffice.

Case 3: Assume $x^{\prime} x \leqslant y y^{\prime}$. In this case, since $y^{\prime} x^{\prime} \in V(x y)$, $(x y) \mathcal{R}(x y)\left(y^{\prime} x^{\prime}\right)=x\left(x^{\prime} x\right)\left(y y^{\prime}\right) x^{\prime}=x\left(x^{\prime} x\right) x^{\prime}=x x^{\prime} \mathcal{R} x$. Thus $x y \mathcal{R} x$ and $x y \in D_{i}$.

Case 4: $x^{\prime} x>y y^{\prime}$. Then $x y \mathcal{L} x^{\prime} x y=x^{\prime} x y y^{\prime} y=y y^{\prime} y=y$, whence $x y \in D_{j} \subseteq D$.

Hence $D$ is closed under multiplication, and clearly each element of $D$ has an inverse in $D$.

In what follows, $\mathcal{B}$ will denote the bicyclic semigroup, $T$ an orthodox semigroup, and $\Phi$ a lattice isomorphism from $\mathcal{L O}(\mathcal{B})$ to $\mathcal{L O}(T)$ Since $E_{\mathcal{B}}$ is isomorphic to $C_{\omega}$, any subset of $E_{B}$ is a regular (inverse) subsemigroup of $\mathcal{B}$. Clearly, then, any subset of $E_{T}$ is an orthodox subsemigroup of $T$. Assume $\mathcal{B}=\left\langle b, b^{-1}\right\rangle$, where $b b^{-1}>b^{-1} b$.

LEMMA 12. If $\mathcal{L O}(\mathcal{B}) \cong \mathcal{L O}(T)$ under the lattice isomorphism $\Phi$, then $T$ is bisimple.

Proof: Suppose $T$ is not bisimple. Take any $\mathcal{D}$-class $D$ of $T$, and set $C=$ $T \backslash D \neq \emptyset$. From Lemma 11 we know that $C$ is an orthodox subsemigroup of $T$. Clearly $C \vee D=T$, and $C \wedge D \subseteq C \cap D=\emptyset$. Thus the inverse proper subsemigroups $C \Phi^{-1}$ and $D \Phi^{-1}$ of $\mathcal{B}$ satisfy $C \Phi^{-1} \vee D \Phi^{-1}=\mathcal{B}$, and $C \Phi^{-1} \cap D \Phi^{-1}=\emptyset$, a contradiction, by Lemma 10 .

The following result of Jones characterises the full inverse subsemigroups of $\mathcal{B}$ (those containing all the idempotents of $\mathcal{B}$ ).

Lemma 13. (Jones [11, Corollary 1.5]). Every non-idempotent full inverse subsemigroup of $\mathcal{B}$ has the form $\left\langle E, b^{-m} b^{m+n}\right\rangle$ for some unique integers $m \geqslant 0, n \geqslant 1$.

THEOREM 14. If $T$ is any orthodox semigroup such that $\mathcal{L O}(\mathcal{B}) \cong \mathcal{L O}(T)$, then $T$ is an inverse semigroup.

Proof: Suppose that $T$ is not inverse. Then there are idempotents $e, f \in E_{T}$ such that $e f \neq f e$. By Lemma 8 , e $\mathcal{R} f$ or $e \mathcal{L} f$; say $e \mathcal{R} f$. We claim that there is an idempotent $g$ of $E_{T}$ which is comparable to $f$, and $g \neq f$. Suppose not. Then for every $g \in E_{T}, f \mathcal{R} g$ or $f \mathcal{L} g$ by Lemma 8, and similarly $e \mathcal{R} g$ or $e \mathcal{L} g$. Since we assumed $e \mathcal{R} f$, the only possibility is $f \mathcal{R} g$. Thus $E_{T}$ is a right-zero semigroup.

Notice that the subgroups in a lattice of orthodox subsemigroups are precisely those elements containing exactly one idempotent. That $\mathcal{B}$ is combinatorial therefore implies that $T$ is combinatorial, and hence $T$ is isomorphic to $E_{T}$. Since $\mathcal{B}$ is not a semilattice, this is a contradiction, and there is an idempotent $g \in E_{T}$ which is comparable to $f$. By Lemma 9, $E_{T}$ is almost commutative, so that $g$ is comparable to $e$ also. Since $T$ is bisimple by Lemma 12, there is an element $a$ of $T$ with inverses $a^{\prime}, a^{*} \in V(a)$ such that $a a^{\prime}=e, a a^{*}=f$, and $a^{\prime} a=a^{*} a=g$. Thus by [2, Lemma 1.31] $\left\langle a, a^{\prime}\right\rangle$ and $\left\langle a, a^{*}\right\rangle$ are bicyclic orthodox subsemigroups of $T$. 
By a result of Jones [11, Theorem 3.1], the bicyclic semigroup is strongly lattice determined within inverse semigroups. Since we assumed that $\mathcal{B}$ is generated by the elements $b$ and $b^{-1}$, then a bicyclic subsemigroup of $\mathcal{B}$ is generated by two elements of the form $b^{-m} b^{m+n}$ and $b^{-m-n} b^{m}$. It follows that $\left\langle a, a^{\prime}\right\rangle \Phi^{-1}=\left\langle b^{-m} b^{m+n}\right\rangle$ and $\left\langle a, a^{*}\right\rangle \Phi^{-1}=\left\langle b^{-k} b^{k+h}\right\rangle$ for some integers $m, k \geqslant 0$ and $n, h \geqslant 1$.

A result of Johnston and Jones [9, Lemma 1.1] states that if $a$ is an element of a full regular subsemigroup $A$ of a regular semigroup $S$, then $V_{S}(a) \subseteq A$. Therefore, $\left\langle a, a^{\prime}\right\rangle \vee E_{T}=\left\langle E_{T}, a, V(a)\right\rangle=\left\langle a, a^{*}\right\rangle \vee E_{T}$. From this we have $\left(\left\langle a, a^{\prime}\right\rangle \vee E_{T}\right) \Phi^{-1}=$ $\left\langle b^{-m} b^{m+n}\right\rangle \vee E_{B}=\left\langle E_{B}, b^{-m} b^{m+n}\right\rangle$, and $\left(\left\langle a, a^{*}\right\rangle \vee E_{T}\right) \Phi^{-1}=\left\langle b^{-k} b^{k+h}\right\rangle \vee E_{B}=$ $\left\langle E_{\mathcal{B}}, b^{-k} b^{k+h}\right\rangle$. Using Lemma $13, b^{-k} b^{k+h}=b^{-m} b^{m+n}$, whence $\left\langle a, a^{\prime}\right\rangle=\left\langle a, a^{*}\right\rangle$, a contradiction.

As was mentioned in the above proof, $\mathcal{B}$ is strongly lattice determined within the class of inverse semigroups. Jones' result [11, Theorem 3.1] is actually stronger, showing that the lattice isomorphism $\Phi$ is induced by a unique isomorphism of $\mathcal{B}$. This yields the following corollaries.

Corollary 15. If $\Phi$ is a lattice isomorphism of $\mathcal{L O}(\mathcal{B})$ onto $\mathcal{L O}(T)$, then $T$ is isomorphic to $\mathcal{B}$ under a unique isomorphism induced by $\Phi$.

Corollary 16. Suppose that $S$ and $T$ are orthodox semigroups, and $\mathcal{L O}(S) \cong$ $\mathcal{L O}(T)$. Then $S$ is completely semisimple if and only if $T$ is.

Proof: $S$ contains a bicyclic subsemigroup if and only if $T$ does.

\section{Simple orthodox SEMigroups}

In this section we show that the class of simple orthodox semigroups which are not completely simple is lattice closed. In addition we obtain results about lattice isomorphisms of some simple inverse semigroups within the class of orthodox semigroups.

Following [9], we shall define a regular semigroup $S$ to be modular [distributive] if and only if its lattice of full regular subsemigroups is modular [distributive]. Clearly, if $S$ and $T$ are orthodox semigroups with $\Phi: \mathcal{L O}(S) \cong \mathcal{L O}(T)$, then $\Phi$ restricts naturally to an isomorphism between the lattices of full orthodox subsemigroups of $S$ and $T$.

REsult 17. [8, Theorem 15]. Take any inverse semigroups $S$ and $T$. If $S$ is a simple modular inverse semigroup which is not a group, and $\Phi$ is a lattice isomorphism from $\mathcal{L O}(S)$ onto $\mathcal{L O}(T)$, then $S$ is isomorphic to $T$ under a unique mapping induced by $\Phi$.

Recall that up to isomorphism the only fundamental simple inverse $\omega$-semigroups are the semigroups $B_{d}(d=1,2,3, \ldots)$. (See [7, V.7].) We can regard $B_{d}$ as an inverse subsemigroup of the bicyclic semigroup $\mathcal{B}=\left\langle b, b^{-1}\right\rangle$, with $d \mathcal{D}$-classes $D_{(0, d)}$, $D_{(1, d+1)}, \ldots, D_{(d-1,2 d-1)}$, where $D_{(i, d+i)}$ is the bicyclic subsemigroup $\left\langle b^{-i} b^{d+i}\right\rangle$. 
THEOREM 18. Take an orthodox semigroup $T$ such that $\mathcal{L O}\left(B_{d}\right) \cong \mathcal{L O}(T)$. Then $T$ is inverse.

Proof: Suppose not. Then there are distinct idempotents $g, h$ of $E_{T}$ such that either $g \mathcal{R} h$ or $g \mathcal{L} h$, say $g \mathcal{R} h$. Now $\{g\} \Phi^{-1}=\{e\}$ and $\{h\} \Phi^{-1}=\{f\}$ for some idempotents $e, f \in B_{d}$; however $e$ and $f$ are comparable, say $e>f$. Since $D_{e}$ is a bicyclic subsemigroup, $D_{e} \Phi$ is bicyclic by Corollary 15. Now $g \in D_{e} \Phi$ so there is an idempotent $k$ in $D_{e} \Phi$ such that $k<g$, and it follows that there is an $a \in T, a^{\prime} \in V(a)$ such that $a a^{\prime}=g$ and $a^{\prime} a=k$.

By Lemma 8, $E_{T}$ is an almost commutative band, and since $k<g \mathcal{R} h$ in $E_{T}$, we also have $k<h$. Now $k \mathcal{L} a \mathcal{R} g \mathcal{R} h$, so we can find $a^{*} \in V(a)$ such that $a a^{*}=h$ and $a^{*} a=k$. Clearly, $\left\langle a, a^{\prime}\right\rangle$ and $\left\langle a, a^{*}\right\rangle$ are bicyclic, so $\left\langle a, a^{\prime}\right\rangle \Phi^{-1}=\left\langle b^{-m} b^{m+n}\right\rangle$ and $\left\langle a, a^{*}\right\rangle \Phi^{-1}=\left\langle b^{-p} b^{p+q}\right\rangle$ for some integers $m, n, p$ and $q$.

We see that $k=a^{*} a=a^{\prime} a \in\left\langle a, a^{\prime}\right\rangle \wedge\left\langle a, a^{*}\right\rangle$, so $\left(\left\langle a, a^{\prime}\right\rangle \wedge\left\langle a, a^{*}\right\rangle\right) \Phi^{-1}=$ $\left\langle a, a^{\prime}\right\rangle \Phi^{-1} \cap\left\langle a, a^{*}\right\rangle \Phi^{-1}=\left\langle b^{-m} b^{m+n}\right\rangle \cap\left\langle b^{-p} b^{p+q}\right\rangle \neq \emptyset$ by Corollary 15. But $b^{-m} b^{m+n} \in$ $D_{(i, d+i)}$ and $b^{-p} b^{p+q} \in D_{(j, d+j)}$ for some $i, j$. Hence $D_{(i, d+i)} \cap D_{(j, d+j)} \supseteq\left\langle b^{-m} b^{m+n}\right\rangle \cap$ $\left\langle b^{-p} b^{p+q}\right\rangle \neq \emptyset$, so $D_{(i, d+i)}=D_{(j, d+j)}$. Now $f \in D_{(i, d+i)}=D_{(j, d+j)} \subseteq D_{e}$ and from this we conclude that $\{g, h\}=\{e, f\} \Phi \subseteq D_{e} \Phi$ which is bicyclic by Corollary 15 , so $g$ and $h$ are comparable, a contradiction.

CoRollary 19. If $\Phi$ is a lattice isomorphism of $\mathcal{L O}\left(B_{d}\right)$ onto $\mathcal{L O}(T)$ for an orthodox semigroup $T$, then $B_{d}$ is isomorphic to $T$ under a unique isomorphism induced by $\Phi$.

Proof: By a result of Jones [10, Proposition 4.1], $B_{d}$ is distributive. The corollary then follows from Result 17.

LEMma 20. Take any lattice-isomorphic orthodox semigroups $S$ and $T$ and any isomorphism $\Phi: \mathcal{L O}(S) \rightarrow \mathcal{L O}(T)$. Let $\alpha: E_{S} \rightarrow E_{T}$ be the bijection induced by $\Phi$. Take any $e, f \in E_{S}$. Then

(i) $e>f \mathcal{D}^{S} e$ if and only if $e \alpha>f \alpha \mathcal{D}^{T} e \alpha$, and

(ii) $e>f \mathcal{J}^{S} e$ if and only if $e \alpha>f \alpha \mathcal{J}^{T} e \alpha$.

Proof: (i) Put $g=e \alpha, h=f \alpha$, and suppose that $e>f \mathcal{D} e$. Then there is an element $a$ in $S$ and $a^{\prime} \in V(a)$ with $a a^{\prime}=e$ and $a^{\prime} a=f$. Then $g, h \in\left\langle a, a^{\prime}\right\rangle \Phi$, which is bicyclic (by Corollary 15, since $\left\langle a, a^{\prime}\right\rangle$ is bicyclic), and thus $g \mathcal{D}^{T} h$ and $g>h$, by the uniqueness of the isomorphism in Corollary 15.

The converse follows, since $\Phi^{-1}$ is a lattice isomorphism of $\mathcal{L O}(T)$ onto $\mathcal{L O}(S)$, and induces the map $\alpha^{-1}$ of $E_{T}$ onto $E_{S}$.

(ii) Assume $e>f \mathcal{J}^{S} e$. A result of Ault [1, Corollary 3.2] implies that if $e$ and $f$ are idempotents in a regular semigroup $S$ with $e>f \mathcal{J}^{S} e$, then there exists a simple $\omega$ subsemigroup $A$ containing $e$ and $f$. Then $A$ is isomorphic to a Bruck-Reilly extension 
of a finite chain of groups $U$, and the Bruck-Reilly extension of the chain of idempotents in $U$ is thus a simple combinatorial $\omega$-subsemigroup, that is, is isomorphic to $B_{d}$, for some $d$.

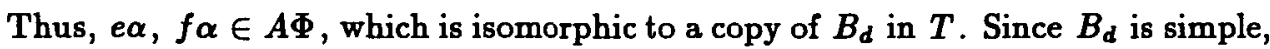
$A \Phi \subseteq J_{e \alpha}$, and then $e \alpha>f \alpha$ by the uniqueness of the isomorphism in Corollary 19 .

For the remainder of this paper, if $S$ and $T$ are orthodox semigroups with $\Phi: \mathcal{L O}(S) \rightarrow \mathcal{L O}(T)$ an isomorphism, we shall denote by $\alpha: E_{S} \rightarrow E_{T}$ the bijection induced by $\Phi$.

Lemma 21. Suppose $T$ is any orthodox semigroup and $S$ is any simple inverse semigroup with $\Phi: \mathcal{L O}(S) \rightarrow \mathcal{L O}(T)$ an isomorphism. Then $T$ is simple.

Proof: Take any $g, h \in E_{T}$ and let $g \alpha^{-1}=e$ and $h \alpha^{-1}=f$. Since $S$ is simple there is an idempotent $k$ of $S$ such that $e \mathcal{D}^{S} k$ and $k<e f \leqslant e$. By Lemma 20, $g=e \alpha \mathcal{D}^{T} k \alpha$ and $k \alpha<e \alpha$. Since $S$ is simple, $k$, ef, e and $f$ are $\mathcal{J}^{S}$-related, so by Lemma $20, k \alpha<(e f) \alpha \leqslant f \alpha$. By [5, Result 5$], T$ is simple.

Lemma 22. Under the hypotheses of Lemma 21, $T$ is inverse.

ProOF: From Lemma 20 if follows that $\alpha$ is an order isomorphism from $\left(E_{S}, \leqslant\right)$ onto $\left(E_{T}, \leqslant\right)$. Take any $g, h \in E_{T}$, and let $e=g \alpha^{-1}, f=h \alpha^{-1}$. If $g$ and $h$ are comparable, then $g h=h g$, so assume $g$ and $h$ are not comparable. Now $e$ and $f$ cannot be comparable, so $\langle e, f\rangle=\{e, f, e f\}$, and thus $\langle e, f\rangle \Phi=(\langle e\rangle \vee\langle f\rangle) \Phi=$ $\langle e\rangle \Phi \vee\langle f\rangle \Phi=\langle g\rangle \vee\langle h\rangle=\langle g, h\rangle$ has three elements, so $g$ and $h$ are not $\mathcal{R}$-or $\mathcal{L}$-related. Since $g h, h g \in\langle g, h\rangle$, checking of several cases shows that $g h=h g$, and hence $T$ is inverse.

If $S$ is a simple inverse semigroup which is not completely simple and $\mathcal{L O}(S)$ is isomorphic to $\mathcal{L O}(T)$, then from Lemma $20, T$ is not completely simple. The following corollary then follows immediately from Result 17 and Lemma 22.

COROLLARY 23. If $S$ is a simple modular inverse semigroup which is not completely simple and $T$ is an orthodox semigroup such that $\Phi: \mathcal{L O}(S) \cong \mathcal{L O}(T)$, then $S$ is isomorphic to $T$ under a unique isomorphism induced by $\Phi$.

Before proving our next result, we consider a useful technical lemma. (The author thanks T.E. Hall for this observation.)

LEMMA 24. A regular semigroup $S$ is simple if and only if any two comparable idempotents are $\mathcal{J}$-related.

PROof: If suffices to show that if any two comparable idempotents of $S$ are $\mathcal{J}$ related, then any two idempotents of $S$ are $\mathcal{J}$-related. Take any $e, f \in E_{S}$. Now $J_{e f} \leqslant J_{f}$, and thus by $\left[4\right.$, Theorem 1] there is an idempotent $g \in J_{e f}$ such that $g \leqslant f$. By hypothesis $J_{g}=J_{f}$, and hence $J_{f}=J_{g}=J_{e f}$. Similarly, $J_{e}=J_{e f}$, so $J_{e}=J_{f}$ as 
desired.

We can now prove one of the main theorems.

Theorem 25. Suppose $S$ and $T$ are orthodox semigroups, and $\Phi$ a lattice isomorphism of $\mathcal{L O}(S)$ onto $\mathcal{L O}(T)$. If $S$ is simple but not completely simple, then $T$ is simple but not completely simple.

Proof: Take any $g, h \in E_{T}$ with $g>h$. Put $e=g \alpha^{-1}$ and $f=h \alpha^{-1}$. If $e$ and $f$ are comparable, then by Lemma 20 we are done, so assume $e$ and $f$ are not comparable. Lemma 8 implies that $e \mathcal{R} f$ or $e \mathcal{L} f$. Since $S$ is not completely simple, then there is an idempotent $k$ of $S$ such that $k<f$. By Lemma 20 and the simplicity of $S, k \alpha<f \alpha=h$ and therefore $k \alpha<g$. Now $\langle e, k\rangle \Phi=\langle g, k \alpha\rangle=\{g, k \alpha\}$, so either $e \mathcal{R} k, e \mathcal{L} k$, or $e$ and $k$ are comparable by Lemma 8. Since $k<f \in V(e)$, checking shows that $k \notin V(e)$, whence $(e, k) \notin \mathcal{R} \cup \mathcal{L}$. Thus $e$ and $k$ are comparable, and by the simplicity of $S$ and Lemma 20 it follows that $k \alpha \mathcal{J}^{T} e \alpha=g$. From $J_{k \alpha} \leqslant J_{h} \leqslant J_{g}=J_{k \alpha}$ we obtain $h \mathcal{J}^{T} g$. From Lemma 24, $T$ is simple.

To see that $T$ is not completely simple, take idempotents $e, f$ of $S$ with $e>f$. Then Lemma 20 gives us $e \alpha>f \alpha$ in $T$, so $T$ is not completely simple.

In contrast to the case of inverse semigroups (see [12, Theorem 2.1]), the assumption that $S$ is not completely simple cannot be dropped. This is evident from Example 7. However, with a small additional hypothesis, we do get a corresponding theorem about completely simple orthodox semigroups.

First we need a result about rectangular bands. We shall call a rectangular band $E$ non-singular if both $\mathcal{R}$ and $\mathcal{L}$ are non-trivial on $E$.

RESULT 26: (Shevrin [14, 15]). If a non-singular rectangular band $E$ is lattice isomorphic under $\Phi$ to a band $F$, then $E$ is isomorphic or antiisomorphic to $F$ under the mapping induced by $\Phi$.

THEOREM 27. Suppose $S$ and $T$ are orthodox semigroups, and $\Phi: \mathcal{L O}(S) \cong$ $\mathcal{L O}(T)$. If $S$ is a completely simple semigroup such that $E_{S}$ is non-singular, then $T$ is completely simple.

Proof: By Result $26, E_{T}$ is a rectangular band; hence $T$ is completely simple.

We shall now derive analogues of Theorems 25 and 27 for 0 -simple semigroups. As was noted in [12], if $\mathcal{L O}(U) \cong \mathcal{L O}(V)$, then $\mathcal{L O}\left(U^{0}\right) \cong \mathcal{L O}\left(V^{1}\right)$. This may happen in the case of 0 -simple semigroups if $S^{*}=S \backslash\{0\}$ is an orthodox subsemigroup of $S$. We shall use the notation $U<V$ to mean that $U$ is an orthodox subsemigroup of $V$. If $L$ is a lattice and $x, y \in L$ with $x \leqslant y$, we shall use the notation $[x, y]$ to denote the interval $\{z \in L: x \leqslant z \leqslant y\}$.

Theorem 28. Suppose $S$ and $T$ are orthodox semigroups which are lattice iso- 
morphic under $\Phi$. Suppose $S$ is 0 -simple, and $E_{S} \backslash\{0\}$ is not a left zero or right zero semigroup. Then either $T$ is 0 -simple, or $S^{*}<S$ and $T=\left(S^{*} \Phi\right)^{1}$ where $S^{*} \Phi$ is simple.

Proof: Notice that since 0 is comparable to every idempotent in $S, 0 \alpha=u$ is either comparable, $\mathcal{R}$-related, or $\mathcal{L}$-related to every idempotent in $T$. We claim that $u$ is comparable to every element of $E_{T}$. For suppose that $u$ is incomparable to $k$ for some $k \in E_{T}$, and let $t=k \alpha^{-1}$. By Lemma $8, u(\mathcal{R} \cup \mathcal{L}) k$, say $u \mathcal{R} k$.

If $S$ is not completely 0 -simple, then there is an idempotent $f<t \mathcal{J}^{S} f$. Let $h=f \alpha$. Since $f>0$, then either $h$ is comparable to $u$ or $h(\mathcal{R} \cup \mathcal{L}) u$ by Lemma 8 . From Lemma 20 we have $h=f \alpha<t \alpha=k J^{T} f \alpha=h$. If $h>u$, then $k>h>u$, which contradicts the hypothesis, so $h(\mathcal{R} \cup \mathcal{L}) u$. Since $h<k, h \notin V(k)=V(u)$. Thus $(h, k) \notin \mathcal{R} \cup \mathcal{L}$, a contradiction.

Now assume that $S$ is completely 0 -simple. Then there exists an $e \in E_{S} \backslash\{0\}$ such that $e \mathcal{D}^{S} t$ and $(e, t) \notin \mathcal{R} \cup \mathcal{L}$ since $E_{S} \backslash\{0\}$ is not a right-zero or left-zero band. Let $g=e \alpha$. Since $e$ and $t$ are incomparable, $g$ and $k$ are incomparable and $(g, h) \notin \mathcal{R} \cup \mathcal{L}$ by Lemma 8 , and by the same lemma, $g$ is comparable to $u$ or $g(\mathcal{R} \cup \mathcal{L}) u$. If $g(\mathcal{R} \cup \mathcal{L}) u$, then $u \mathcal{R} k$ and $(g, k) \notin \mathcal{R} \cup \mathcal{L}$ implies $g \mathcal{L} u$. Then $V(u)$ is a non-singular rectangular band, and by Result $26, V(0)$ is a non-singular rectangular band in $E_{S}$, a contradiction.

The only other possibility is that $g$ is comparable to $u$. If et $\in R_{e} \cap L_{t}$, then $V(t)$ is a non-singular rectangular band, and hence so is $V(t \alpha)=V(k)=V(u)$, which is a contradiction. Thus $H=R_{e} \cap L_{t}$ is not a group, and $H^{2}=\{0\}$. Let $a \in H$ and $a^{\prime}$ the inverse of $a$ in $R_{t} \cap L_{e}$. Then the subsemigroup $\left\langle a, a^{\prime}\right\rangle=\left\{a, a^{\prime}, e, t, 0\right\} \in \mathcal{L O}(S)$, and note that $e t=t e=0$. Let $B=\left\langle a, a^{\prime}\right\rangle \Phi$ and take any $b \in B \backslash E_{T}$ and $b^{\prime} \in V(b) \cap B$. The subsemigroup $\left\langle b, b^{\prime}\right\rangle$ is in $\mathcal{L O}(T)$. Since $\left\langle a, a^{\prime}\right\rangle$ covers precisely the orthodox subsemigroup $\{e, t, 0\}$ and contains no non-trivial groups, then $B$ covers precisely $\{e \alpha, t \alpha, 0 \alpha\}=\{g, k, u\}$, so $\left\langle b, b^{\prime}\right\rangle=B$. Also $b b^{\prime} \neq b^{\prime} b$ since $B$ contains no nontrivial subgroups. Hence $\left\{b b^{\prime}, b^{\prime} b\right\}=\{g, u\}$ or $\{g, k\}$ or $\{u, k\}$. If $\left\{b b^{\prime}, b^{\prime} b\right\}=\{g, u\}$, then $B$ contains the bicyclic semigroup, and hence so does $\left\langle a, a^{\prime}\right\rangle$, a contradiction. If $\left\{b b^{\prime}, b^{\prime} b\right\}=\{g, k\}$, then $g \mathcal{D}^{T} k \mathcal{R} u$, and since $g$ is comparable to $u,\{g, u\}$ is contained in a bicyclic semigroup, and by Corollary 15 , so is $\{e, 0\}$ in $S$, again a contradiction.

So $\left\{b b^{\prime}, b^{\prime} b\right\}=\{u, k\}$, say $b b^{\prime}=u$ and $b^{\prime} b=k$, whence we have $b \mathcal{H}^{B} k$. Hence $b=k$, which is not possible. We conclude therefore that $u$ is comparable to every idempotent in $T$.

It now follows that $\{u\}$ is a $\mathcal{J}^{T}$-class. For if there is another idempotent $g$, say, in $J_{u}$, then since $u$ is comparable to $g$, by Lemma $20 u \alpha^{-1}=0 \mathcal{J}^{S} g \alpha^{-1}$.

Next we shall prove that any two comparable idempotents of $T \backslash\{u\}$ are $\mathcal{J}^{T}$. related. Suppose that $g, h \in E_{T} \backslash\{u\}$, and $g>h$. Let $e=g \alpha^{-1}, f=h \alpha^{-1}$. From 
Lemma 8 we have $e$ is comparable to $f$ or $e(\mathcal{R} \cup \mathcal{L}) f$. If $e$ is comparable to $f$, then by Lemma 20, $g \mathcal{J}^{T} h$. So now assume $e(\mathcal{R} \cup \mathcal{L}) f$, say $e \mathcal{R} f$. (The case $e \mathcal{L} f$ will follow in a similar fashion.) We consider two cases.

Case 1: $S$ is not completely 0 -simple.

Then there is an idempotent $k$ with $f>k>0$. By Lemma $20, h>k \alpha \mathcal{J}^{T} h$, so $g>k \alpha$. Hence $e$ and $k$ are comparable or $e(\mathcal{R} \cup \mathcal{L}) k$ by Lemma 8. Since $f>k$, the latter is impossible. Again by Lemma $20, g \mathcal{J}^{T} k \alpha$ and so $g \mathcal{J}^{T} h$.

Case 2: $S$ is completely 0 -simple.

If $e$ and $f$ are contained in a non-singular rectangular band, then by Result 26, $g$ and $h$ are contained in a non-singular rectangular band, and hence $g \mathcal{J}^{T} h$. If $e$ and $f$ are not contained in a non-singular rectangular band, then since $E_{S} \backslash\{0\}$ is not a left group or a right group, there is an idempotent $k \neq 0$ with $k \notin V(e)$. Let $a \in R_{e} \cap L_{k}$, and let $a^{\prime}, a^{*}$ be inverses of $a$ with $a^{\prime} \in R_{k} \cap L_{f}, a^{*} \in R_{k} \cap L_{e}$. Then $a^{2}=0$, and $\left\langle a, a^{\prime}\right\rangle=\left\{a, a^{\prime}, f, k, 0\right\}$ and $\left\langle a, a^{*}\right\rangle=\left\{a, a^{*}, e, k, 0\right\}$. Hence $\left\langle a, a^{\prime}\right\rangle$ covers precisely the orthodox subsemigroup $\{e, k, 0\},\left\langle a, a^{*}\right\rangle$ covers precisely the orthodox subsemigroup $\{f, k, 0\}$, and neither $\left\langle a, a^{\prime}\right\rangle$ nor $\left\langle a, a^{*}\right\rangle$ contains any nontrivial groups. As earlier in the proof, $\left\langle a, a^{\prime}\right\rangle \Phi=\left\langle b, b^{\prime}\right\rangle,\left\langle a, a^{*}\right\rangle \Phi=\left\langle c, c^{\prime}\right\rangle$ for some $b, c \in S \Phi, b^{\prime} \in V(b)$ and $c^{\prime} \in V(c)$. We see that $\left\{b b^{\prime}, b^{\prime} b\right\}=\{g, k \alpha\},\{k \alpha, u\}$ or $\{g, u\}$. The last two possibilities can be eliminated since $\left\langle b, b^{\prime}\right\rangle$ is not bicyclic, so $\left\{b b^{\prime}, b^{\prime} b\right\}=\{g, k \alpha\}$, and similarly $\left\{c c^{\prime}, c^{\prime} c\right\}=\{h, k \alpha\}$, and it follows that $g \mathcal{D}^{T} h$. This completes Case 2.

It now follows that any two comparable non- $u$ idempotents of $T$ are $\mathcal{J}^{T}$-related, and thus either $J_{u} \geqslant J_{a}$ for all $a \in T$, or $J_{u} \leqslant J_{a}$ for all $a \in T$. In the first case, then there is only one $\mathcal{J}^{T}$-class below $J_{u}$, for if $e, f \in E_{T} \backslash\{u\}$, then $J_{u}>J_{e} \geqslant J_{e f} \leqslant$ $J_{f}<J_{u}$, and by [4, Theorem 1], $J_{e}=J_{e f}=J_{f}$. In this case we claim that $u$ is the identity for $T$. Take any $x \in T$ and any $x^{\prime} \in V(x)$. Then $u \geqslant x x^{\prime}$, so $u x x^{\prime}=x x^{\prime}$ and $u x=x$. Similarly $x u=x$. Now $T \backslash\{u\}$ is an orthodox subsemigroup of $T$, so $S^{*}<S, S^{*} \Phi=T \backslash\{u\}$, and $S^{*} \Phi$ is simple by Lemma 24 .

Suppose $J_{u} \leqslant J_{a}$ for all $a \in T$. Then $u$ is the zero of $T$. Take any $g, h \in E_{T} \backslash\{u\}$. If $g h \neq u$, then $g \geqslant g h g \mathcal{J}^{T} h g h \leqslant h$, and by the above argument, $g \mathcal{J}^{T} g h g$ and $h \mathcal{J}^{T} h g h$, whence $g \mathcal{J}^{T} h$. So assume $g h=u$, and let $e=g \alpha^{-1}$ and $f=h \alpha^{-1}$. Since $\langle g, h\rangle=\{g, h, u\},\langle e, f\rangle=\{e, f, 0\}$, and so $e f=f e=0$. Since $S$ is 0 -simple, there is a non-zero idempotent $k$ in $S$ such that $e \mathcal{D}^{S} k \leqslant f$, and clearly $e k=0$. Choose $a \in R_{e} \cap L_{k}$ and an inverse $a^{\prime} \in R_{k} \cap L_{e}$. Then $\left\langle a, a^{\prime}\right\rangle=\left\{a, a^{\prime}, e, k, 0\right\}$, and as in previous arguments, $\left\langle a, a^{\prime}\right\rangle \Phi=\left\langle b, b^{\prime}\right\rangle$, where $\left\{b b^{\prime}, b^{\prime} b\right\} \subseteq\{g, h, u\}$, and since $\left\langle b, b^{\prime}\right\rangle$ is not bicyclic, $\left\{b b^{\prime}, b^{\prime} b\right\}=\{g, h\}$, so that $g \mathcal{D}^{T} h$. Thus there is only one non- $\{u\} \mathcal{J}^{T}$-class, and we conclude that $T$ is 0 -simple.

It is not possible to omit the hypothesis that $E_{S} \backslash\{0\}$ is not left zero or right zero, 
as the next example shows.

EXAmple 29. Let $S$ consist of two $\mathcal{R}$-related idempotents, $e$ and $f$, with zero adjoined. Let $T$ be the three-element chain $g>h>0$. Then $\mathcal{L O}(S)$ and $\mathcal{L O}(T)$ are isomorphic as shown below, $S$ is 0 -simple, but $T$ contains three $\mathcal{J}^{T}$-classes.

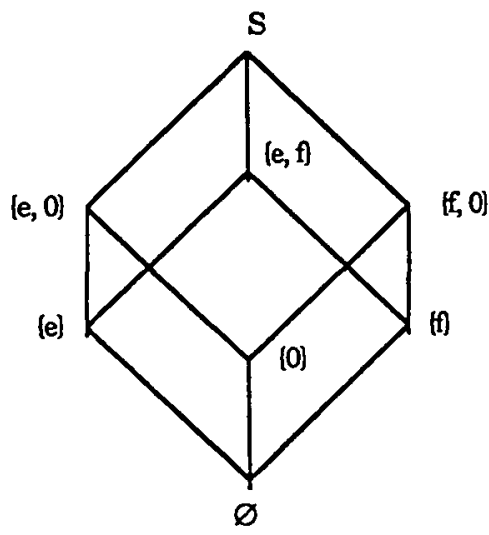

cas)

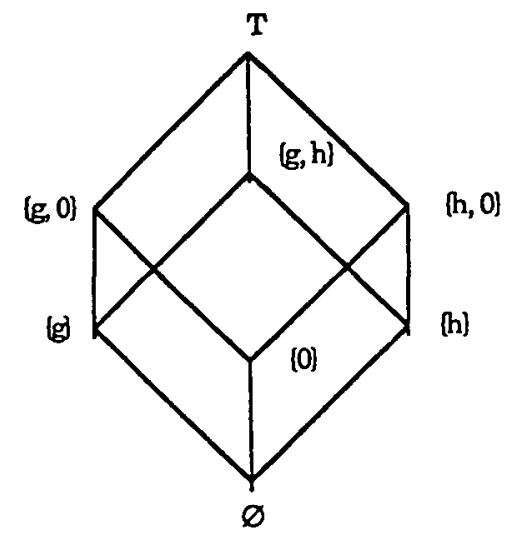

LOT)

The next theorem shows that the situation cannot get much worse than this, however.

Theorem 30. Suppose $S$ is a right group or left group (possibly with zero adjoined), and $T$ is an orthodox semigroup with $\Phi: \mathcal{L O}(S) \cong \mathcal{L O}(T)$. Then $T$ is a chain of left groups and right groups.

Proof: Suppose $S$ is a right group or left group (with zero adjoined). By Result 26, $T$ contains no non-singular rectangular bands. No two $\mathcal{J}^{T}$-related idempotents are comparable by Lemma 20, and since any two idempotents of $T$ are either comparable or $\mathcal{R} \cup \mathcal{L}$-related by Lemma 8 , any two $\mathcal{J}^{T}$-related idempotents are therefore all $\mathcal{R}$-related or all $\mathcal{L}$-related. Suppose we take two distinct $\mathcal{J}^{T}$-classes, say $J_{g}$ and $J_{h}$ containing idempotents $g$ and $h$, respectively. Then $g$ and $h$ are comparable since they are not $\mathcal{R} \cup \mathcal{L}$-related, and thus $J_{g}$ and $J_{h}$ are comparable. Hence, each $\mathcal{J}^{T}$-class is a left group or a right group, and the $\mathcal{J}^{\boldsymbol{T}}$-classes form a chain.

\section{REFERENCES}

[1] J.E. Ault, 'Semigroups with bisimple and simple $\omega$-subsemigroups', Semigroup Forum 9 (1975), 318-333.

[2] A.H. Clifford and G.B. Preston, Algebraic theory of semigroups, Math. Surveys 7 (Amer. Math. Soc., Providence, R.I., Vol. I, 1961, Vol. II, 1967).

[3] P. Crawley and R.P. Dilworth, Algebraic theory of lattices (Prentice-Hall, Englewood Cliffs, N.J., 1973). 
[4] T.E. Hall, 'On the natural ordering of $\mathcal{J}$-classes and of idempotents in a regular semigroup', Glasgow Math. J. 11 (1970), 167-168.

[5] T.E. Hall, 'On regular semigroups', J. Algebra 24 (1973), 1-24.

[6] T.E. Hall, 'Almost commutative bands', Glasgow Math. J. 13 (1972), 176-178.

[7] J.M. Howie, An introduction to semigroup theory (Academic Press, London, 1976).

[8] K.G. Johnston, 'Lattice isomorphisms of modular inverse semigroups', Proc. Edinburgh Math. Soc. 31 (1988), 441-446.

[9] K.G. Johnston and P.R. Jones, 'The lattice of full regular subsemigroups of a regular semigroup', Proc. Royal Soc. Edinburgh 98A (1984), 203-214.

[10] P.R. Jones, 'Distributive inverse semigroups', J. London Math. Soc. 17 (1978), 457-466.

[11] P.R. Jones, 'Lattice isomorphisms of distributive inverse semigroups', Quart. J. Math. Oxford Ser. 30 (1979), 301-314.

[12] P.R. Jones, 'Inverse semigroups determined by their lattices of inverse subsemigroups', $J$. Austral. Math. Soc. (Ser. A) 30 (1981), 321-346.

[13] N.R. Reilly and H.E. Scheiblich, 'Congruences on regular semigroups', Pacific J. Math. 23 (1967), 349-360.

[14] L.N. Shevrin, 'Lattice properties of idempotent semigroups I', Siberian Math. J. 6 (1965), 459-474. (in Russian).

[15] L.N. Shevrin and A.J. Ovsyannikov, 'Semigroups and their subsemigroup lattices', Semigroup Forum 27 (1983), 1-154.

[16] M. Suzuki, Structure of a group and the structure of its lattice of subgroups (SpringerVerlag, Berlin, Heidelberg, New York, 1956).

Department of Mathematics

Royal Melbourne Institute of Technology

P.O. Box $2476 \mathrm{~V}$

Melbourne, Vic. 3001

Australia 